\title{
Visualizing the Main Monuments of Crete through an Abstract Interactive 3D Model
}

\author{
Panagiotis Parthenios \\ Technical University of Crete \\ 127 El. Venizelou Street \\ 73100 Chania \\ Greece \\ panos@parthenios.com
}

\begin{abstract}
Conceptualization of information allows improved visualization and manipulation of large amounts of data. Especially in digitization of cultural heritage, and when aiming at presenting more than one monument, abstraction of information becomes the key solution. Our proposed application aims to present the main archaeological monuments of Crete through a conceptual 3D model. It allows the user to navigate among all the main monuments and approach them gradually in different levels of detail. Furthermore, the ability to switch between the seven historical periods offers a comparative study of their evolution in time. By providing the minimum needed information at each level of abstraction, "levels of detail", we can create an online platform, light and easy to use.
\end{abstract}

Visualizing information. Abstract. 3D model. Conceptual. Interactive.

\section{INTRODUCTION}

This paper aims to present the work in progress our research team has been carrying out on designing a conceptual 3D model of the main historical monuments of Crete throughout time. We are working on creating an online platform that would be open to the public and would aim to promote the cultural heritage of Crete, through a simple, userfriendly, intuitive environment. We have just started implementing the Conceptual Modeling Language (ConML) as a tool to handle and visualize the large amounts of information that the long list of monuments entails.

\section{CONCEPTUAL DIAGRAM}

Crete is the largest island of Greece, located to the south, famous for its rich cultural history, which dates back to the Middle Paleolithic age, 128,000 BC. Crete was the center of the Minoan civilization (2,700-1,420 BC). Since then, a large number of monuments has been documented throughout the different historical periods, the most important of which are the following seven (7):

$\begin{array}{ll}\text { 1. } & \text { Minoan } \\ \text { 2. } & \text { Hellenistic } \\ \text { 3. } & \text { Roman } \\ \text { 4. } & \text { Byzantine } \\ \text { 5. } & \text { Venetian }\end{array}$

6. Ottoman

7. Modern

Our prime challenge has been how to manage such a large amount of information over the internet, in a transparent, light and simple way for the end user, in addition to offering the ability to compare data over time, during the historical periods. In order to achieve this we have been using the notion of Conceptual Modeling along with the principles of Model Based Information and Object Oriented Databases. The idea is simple: instead of having all information to its full extend available up front, we break it into nodes, levels of abstraction, called "Levels of Detail", providing the minimum information needed at each given time. Information is stored on each object, each monument, along with its different Levels of Detail. The Levels of Detail that we are using in this platform are the following five (5):
a. Prefectures
b. Cultural provinces
c. Settlements - Towns
d. Building complexes
e. Buildings - Monuments

The conceptual diagram on which this platform is based can be seen in Figure 1. 


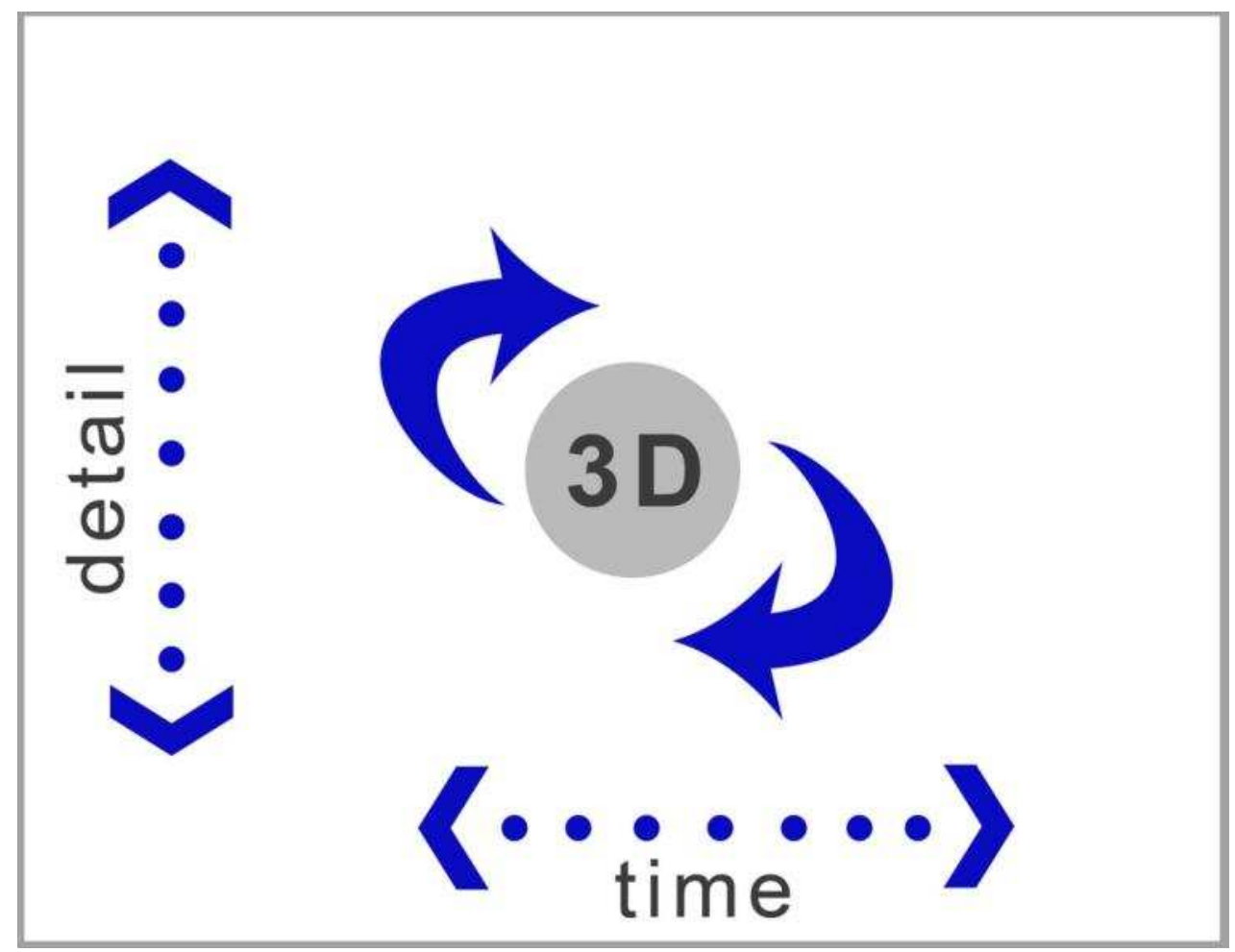

Figure 1: Conceptual diagram of the platform.

\section{PLATFORM}

The platform is comprised of the main, central space, where the 3D models are presented, and two scrollable sidebars, one horizontal and one vertical. The horizontal one controls time, and allows the user to switch between the seven historical periods and the vertical one controls the level of detail, allowing the user to switch between more or fewer abstract modes. At the same time, the user has the ability to navigate in real time in the main space around the models, using pan, zoom in/out, rotate, etc. Only when the user reaches the fifth Level of Detail, that of a single monument, he/she has access to all the available related information, which depending on the type of monument could be:

\footnotetext{
- $\quad$ Photographs

- Architectural drawings (of the existing and/or the restored monument)

- 3D model (of the existing and/or the restored monument)

- Walkthrough animation (of the existing

and/or the restored monument)

- $\quad$ Video of the area as it is today

- $\quad$ Maps of the area

- $\quad$ Related documents with more information

- $\quad$ Related links to other websites

- $\quad$ Keywords. Use of keywords allows a crossreference function independent of the 3D models.
}

The philosophy of the monument presentation is intended to address mainly non-experts, therefore it follows a more abstract and simplified view of information. It should be easy to use for a visitor who does not have a deep knowledge about Crete and its civilization and would like to be informed at a glance what to visit and where. On a second level, the visitor can focus more on a group of monuments and prepare for his/her visit acquiring more specific information, stored "on" the monument's model itself. The application is currently based on Adobe Flash in order to provide maximum compatibility with most of the major web browsers, to be light and easy to use and to avoid installation of other software. At the same time we are investigating whether technologies such as Unity 3D or SpiderGL can provide as a more suitable environment to work with.

The proposed application could take advantage of other related research projects which have rigorously documented and categorized the monuments of Crete, such as the "Digital Crete: Mediterranean Cultural Itineries" (http://digitalcrete.ims.forth.gr), which was implemented under the framework of the Greek Operational Program Information Society (Action 1: Education and Culture, Measure 1.3: Documentation, Management \& Promotion of Greek Cultural Heritage) (http://www.infosociety.gr). 

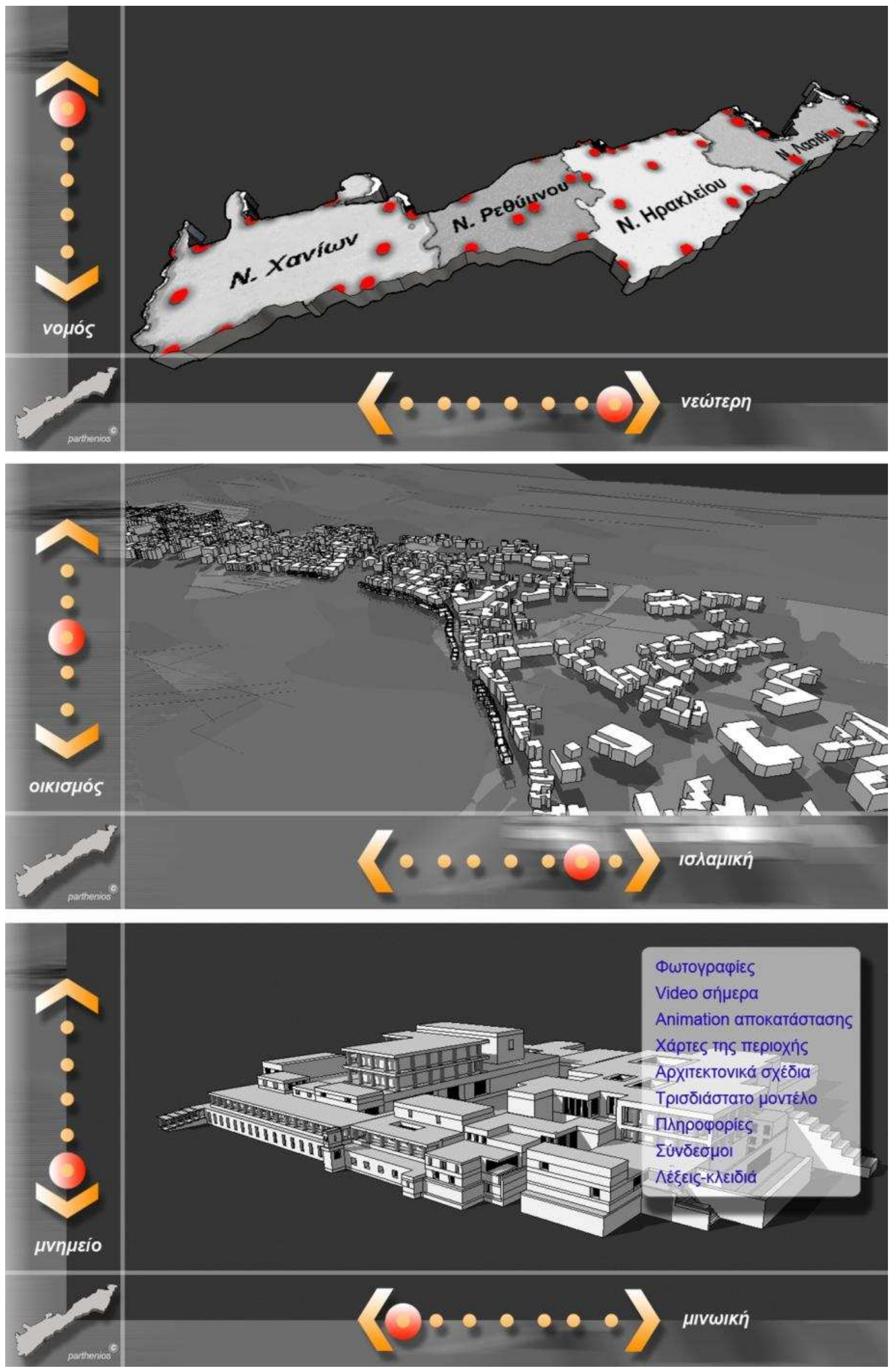

Figure 2: Three modes of the application's interface. 


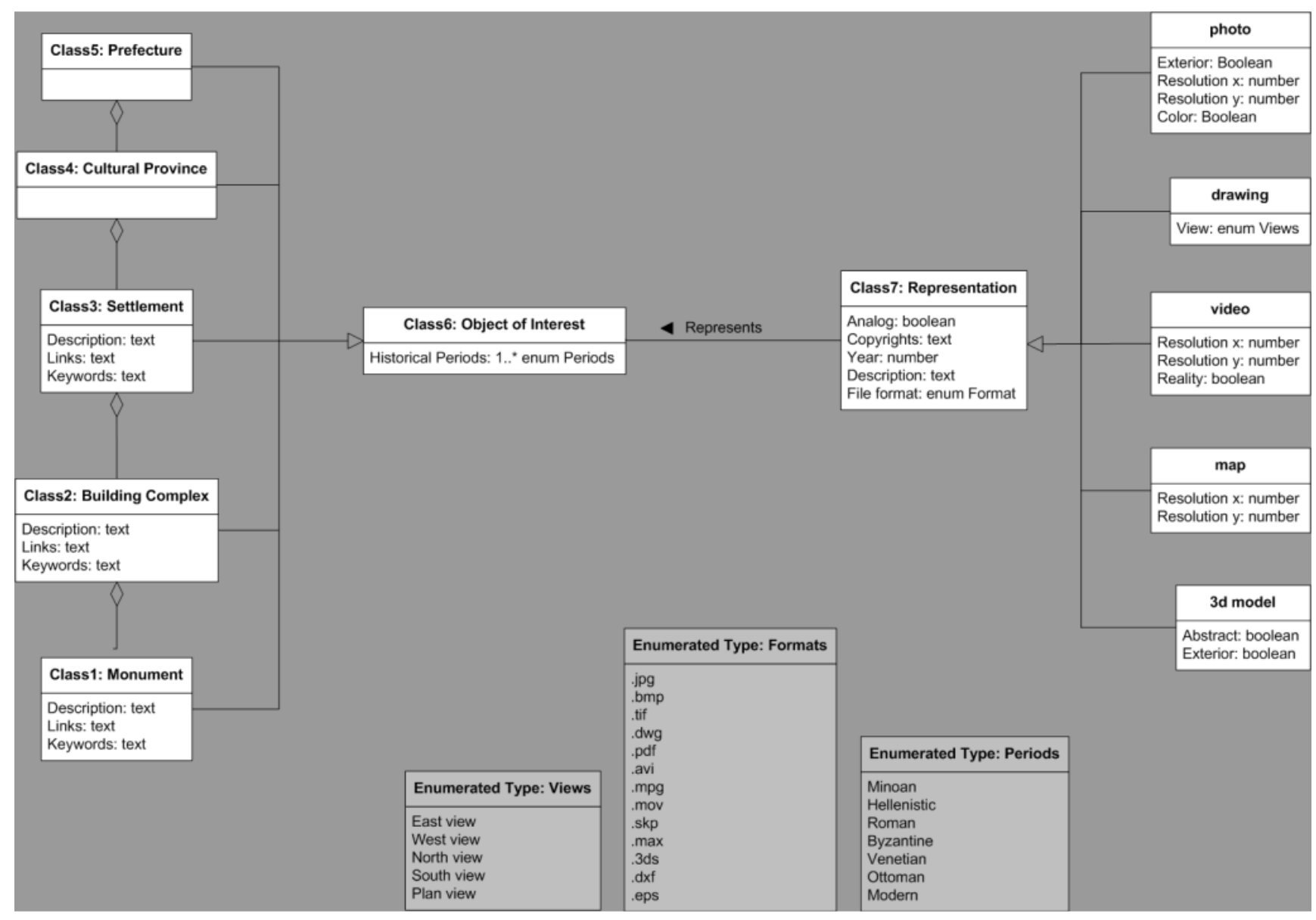

Figure 3: Conceptual model using ConML.

\section{CONCLUSIONS}

The primary contribution of the proposed platform is the ability to capture time in a comparative format. Nevertheless, the fourth dimension is exploited here in a more abstract way than other scientific approaches (e.g., Kulitz, Ferschin \& Matejowsky 2010) since realism and full detailing is not the goal of this application. Furthermore, its advantages are: the user friendly interface which is addressed towards non experts and its ability to continuously expand with new material regarding either new monuments or new information for existing monuments. Some of the issues we are currently working on are:

- Subjectivity due to abstraction. When information is abstracted, the role of the person who decides which information should be secluded is a key role since it could possibly skew the end result.

- $\quad$ Use of multiple semantic links.

- Interoperability / expansion. The proposed application could serve as a central platform which could be joined by other applications which focus on a more detailed, photorealistic monument representation.
- $\quad$ Building Information Modeling (BIM). What can we learn from the structure of information used today in BIM?

- $\quad$ Conceptual Modeling Language (ConML). ConML has many advantages in organizing large amounts of information, especially when dealing with cultural heritage. How useful can such a conceptual model prove to be?

\section{REFERENCES}

Digital Crete: Mediterranean Cultural Itineries http://digitalcrete.ims.forth.gr (retrieved 28 June 2011).

\section{Conceptual Modeling Language}

http://www.conml.org (retrieved 28 June 2011).

\section{SpiderGL}

http://spidergl.org

(retrieved 14 March 2012).

Kulitz, I., Ferschin, P., and Matejowsky, P. (2010) 4D Visualization Methods in Archaeology. 15th International Conference on Cultural Heritage and New Technologies, Vienna, Austria, November 2010. Museen der Stadt Wien, Vienna. 